\title{
Can We Study the Correlation between Human Brain Signal and Other Biological Signals?
}

\author{
Hamidreza Namazi \\ 167 Division Ave Brooklyn, New York 11211, USA
}

*Corresponding Author: Hamidreza Namazi, 167 Division Ave Brooklyn, New York 11211, USA, E-mail;hrnneuromap@gmail.com

Received: July 04, 2017

Accepted: July 06, 2017
Published: July 07, 2017
The brain as the main unit of nervous system controls all organs of human body. Human receives different internal and external stimuli that most of them are controlled by the brain. Different organs of body are engaged in receiving of these stimuli. For instance, eyes receive visual stimuli and send the message to the brain for further processing. Our respiration system receives olfactory stimuli, which accordingly processes by the brain. In case the stimulus is bigger than the threshold stimulus, the brain will react and send the feedback message to the organ for the proper action. For instance, in case we sniff a bad smell, the brain considers it as unwanted stimulus and send message to our respiration system to refuse sniffing. In fact, the stimuli are crucial to the survival of organisms and often manifests as alterations in the structure and function of the nervous system [1].

The brain reaction to different types of stimuli can be analyzed using brain signal, which is called Electroencephalogram (EEG) signal. On the other hand, the response of several organs to different types of stimuli can be detected using their related signals. The reaction of eye during watching a visual stimulus can be measured using eye movement signal. The respiration activity due to smelling different olfactory stimuli can be measured using respiration signal. Electromyography (EMG) is an electrodiagnostic medicine technique for evaluating and recording the electrical activity produced by skeletal muscles.

Since the brain controls the human body through the nervous system, thus the brain response to different stimuli should be related to different organs' feedback to those stimuli [2]. Now, the question, which arises here, is that how we can investigate the relation between brain feedback and organs' feedback to stimuli?

During many years, different methods have been developed to analyze different types of bio signals. Some of these methods showed their ability to analyze the brain signals (EEG), and some of the mare important to analyze other types of bio signals [3].

One useful approach to study the scaling properties of many biological time series is to apply methods derived from the concept of selfsimilar (fractal) processes [4-6]. A fractal is a natural phenomenon or a mathematical set that exhibits a repeating pattern that displays at every scale [7]. The class of regular fractals includes many familiar simple objects, such as line intervals, solid squares, and solid cubes, and many irregular objects [8]. The scaling rules are characterized by "scaling exponents" (dimension). "Simple" regular fractals have integer scaling dimensions [9]. Complex selfsimilar objects have non-integer dimension [10]. In fact, two main features, which are discussed in case of analysis of fractal time series, are fractal dimension and the Hurst exponent.

Fractal theory has been used widely in analysis of EEG signals. For instance, in [11] we showed that complexity and entropy of the EEG signal is coupled with the molecular complexity and entropy of the odorant, where more structurally complex odorants have greater effect on the EEG signal. In another work [12], we found the correlation between the variations of the Hurst exponent for the EEG signal and the applied auditory stimuli to subjects. See also [13-14].

On the other hand, many works have been reported in literature, which analyze other types of bio signals using fractal theory. For instance, 
in a recent work [15] we analyzed the influence of fractal structure of visual stimuli on fractal dynamics of fixational eye movements. We showed that fractal dynamics of eye movements is correlated with the fractal structure of visual stimuli presented to subjects.In another work [16], we investigated the effect of complexity of several olfactory stimuli (odorants) on fractal dynamics of human respiration signal. We found out that respiration signal gets less complex by choosing odorants with higher molecular complexity. In addition, the works on analyzing heart rate [17] and human stride time series [18] are noteworthy to mention.

Based on the mentioned works, EEG signal and other type of bio-signal shows their feedback to external stimuli by variations of their fractal dimension. Now, the hypothesis that we bring here is that the fractal nature of these signal should be related. In fact, using this way we can easily investigate about the correlation between brain feedback and other organs' feedback to external stimuli. For instance, we can analyze the simultaneous variations of fractal dimension for EEG signal and heart rate signal due to olfactory stimuli. Accordingly, we can discuss about the variation of complexity and memory within these types of signals, which leads us to link all organs' reactions as a general map for human body.

\section{REFERENCES}

[1] Namazi H, Kulish VV. A mathematical based calculation of a myelinated segment in axons. Comput. Biol. Med. 2013 Jul; 43(6):693-8. doi: 10.1016/j.compbiomed.2013.03.005.

[2] Seetharaman, K., Namazi, H. \&Kulsih, V. V. Phase lagging model of brain response to external stimuli-modeling of single action potential. Comput. Biol. Med. 42, 857-862 (2012).

[3] Namazi, H. \&Kulish V. V.Mathematical Modeling of Human Brain Neuronal Activity in the Absence of External Stimuli. Journal of Medical Imaging and Health Informatics 2(4):400-407 (2012).

[4] HamidrezaNamazi, Vladimir V. Kulish, FatemehDelaviz, Ali Delaviz. Diagnosis of skin cancer by correlation and complexity analyses of damaged DNA. Oncotarget, Advance Publications 2015. DOI: 10.18632/oncotarget.6003

[5] Namazi, H. et al. Mathematical Modelling and Prediction of the Effect of Chemotherapy on Cancer Cells. Sci. Rep. 5, 13583; doi: 10.1038/srep13583 (2015).
[6] Namazi, H. \&Kiminezhadmalaie, M. Diagnosis of Lung Cancer by Fractal Analysis of Damaged DNA. Comput. Math. Methods Med. 2015; 1-13 (2015).

[7] HamidrezaNamazi, Can We Correlate the Spider's Brain Activity to it Spinning Web Activity?. ARC Journal of Neuroscience. 2017; 2(1):17-18. doi:dx.doi.org/10.20431/2456057X.0201005.

[8] Namazi, H. et al. The fractal based analysis of human face and DNA variations during aging. Biosci. Trends. 10, doi: 10.5582/bst.2016.01182 (2016).

[9] Namazi H, Akrami A, Haghighi R, Delaviz A, Kulish V.V. Analysis of the Influence of Element's Entropy on the Bulk Metallic Glass (BMG) Entropy, Complexity and Strength. Metallurgical and Materials Transactions A.DOI: 10.1007/s11661-016-3870-3 (2016).

[10] HamidrezaNamazi, Can We Explain the Memory Transfer between Generations by Mathematical Analysis of DNA Walk? ARC Journal of Neuroscience. 2017; 2(2):1-3. doi:dx.doi.org/10.20431/2456- 057X.0202001.

[11] Namazi, H., Akrami, A., Nazeri, S. \&Kulish, V. V. Analysis of the Influence of Complexity and Entropy of Odorant on Fractal Dynamics and Entropy of EEG Signal. BioMed. Res. Int. 2016, 5469587, doi: 10.1155/2016/5469587 (2016).

[12] Namazi, H. et al. Analysis of the influence of memory content of auditory stimuli on the memory content of EEG signal. Oncotarget. 7(35):56120-56128.

doi: 10.18632/oncotarget.11234 (2016).

[13] Namazi, et al. A signal processing based analysis and prediction of seizure onset in patients with epilepsy. Oncotarget 7, 342-350; DOI: 10.18632/oncotarget.6341 (2016).

[14] HamidrezaNamazi and Vladimir V. Kulish, Fractional Diffusion Based Modelling and Prediction of Human Brain Response to External Stimuli. Computational and Mathematical Methods in Medicine, 2015, doi:10.1155/2015/148534 (2015).

[15] Namazi, H. Kulish, V. V. \&Akrami, A. The analysis of the influence of fractal structure of stimuli on fractal dynamics in fixational eye movements and EEG signal. Sci. Rep. 6, 26639; DOI: 10.1038/srep26639 (2016)

[16] Namazi, H., Akrami, A. \&Kulish, V. V. The Analysis of the Influence of Odorant's Complexity on Fractal Dynamics of Human Respiration. Sci. Rep. 6, 26948; DOI: 10.1038/srep26948 (2016).

[17] Namazi, H. and Kulish, V. V. Fractal Based Analysis of the Influence of Odorants on Heart Activity. Sci. Rep. 6, 38555; doi: 10.1038/srep38555 (2016). 
[18] Namazi, H. \&Kulish, V. V. Mathematicalbased modeling and prediction of the effect of external stimuli on human gait. Int. J. Numer.
Method Biomed. Eng. 32; DOI: 10.1002/cnm.2805 (2016).

Citation: Hamidreza Namazi, Can We Study the Correlation between Human Brain Signal and Otherbiological Signals?. ARC Journal of Neuroscience. 2017; 2(2):7-9. doi:dx.doi.org/10.20431/2456057X.0202003.

Copyright: (C) 2017 Authors. This is an open-access article distributed under the terms of the Creative Commons Attribution License, which permits unrestricted use, distribution, and reproduction in any medium, provided the original author and source are credited. 